\title{
A Morphological Analysis Method for Geological Bodies and Its Application on Three-dimensional Visualization Environment
}

\author{
Yanhong Zou ${ }^{\mathrm{a}, \mathrm{b}}$, Xiancheng $\mathrm{Mao}^{\mathrm{a}, \mathrm{b}}$,Wenfeng $\mathrm{Xi}^{\mathrm{a}}$ \\ ${ }^{a}$ School of Geoscience and Info-physics Central South University Changsha, Hunan Province, 410083, China \\ ${ }^{b}$ Key Laboratory of Metallogenic Prediction of Nonferrous Metals, Ministry of Education Central South \\ University Changsha, Hunan Province, 410083, China
}

\begin{abstract}
Aiming at the spatial shape analysis of complex geological bodies in stereoscopic quantitative prediction of concealed ore bodies, a three-dimensional morphological analysis method for geological bodies based on 3-dimensional raster model under visualization environment was put forward by combining mathematical morphology with Euclidean distance transform theory. Firstly, the 3-dimensional visualization models for geological bodies were constructed on the basis of the 3-dimensional geological modeling (3DGM) technology; Secondly, the algorithm for extracting the surface shape trend of geological body with the 3-dimensional raster model was proposed by using mathematical morphology filtering. By the combination of morphological filtering, global set operation and three-dimensional Euclidean distance transform, the models for the quantitative analysis and extraction of the shape undulance were established. Lastly, as a case study, the three-dimensional morphological analysis method was applied in analyzing quantitatively the Xinwuli magmatic body in Fenghuangshan ore field in Tongling, Anhui Province. By means of the calculation model of Euclidean distance field, the quantitative extraction of the shape trend and shape undulance as the quantitative indexes of geological ore-controlling factors were achieved after building the 3D raster models of the magmatic body. The results show that the morphological analysis method is feasible to calculate various morphological parameters of complex geological bodies and extract quantitative indexes of geological ore-controlling factors successfully for stereoscopic quantitative predication of concealed ore bodies.
\end{abstract}

Index Terms: Geological body; 3-dimensional visualization; morphological analysis; mathematical morphology; Euclidean distance transform

(C) 2012 Published by MECS Publisher. Selection and/or peer review under responsibility of the Research Association of Modern Education and Computer Science.

\section{Introduction}

The formation of a mineral deposit has relation with various geological actions. The geological bodies such

* Corresponding author.

E-mail address: z-yhong@163.com,xcmao@126.com,you-xiwenfeng@163.com 
as Magmatic rock, strata, faults and folding structures are often the main ore-forming factors in the deposits. The shape trend of geological body reflects its major characteristics in the space, while the shape undulance reflects that of local in a certain spatial direction. In the deposit, the shape trend and the shape undulance of the geological body always have an important impact on the ore-formation actions. Hence, with the development of the quantitative prediction of mineral resources in three-dimension space [1-2], three-dimensional geological modeling and spatial analysis technology are applied to depict the geological bodies and extract the ore-controlling geological indexes quantitatively.

Currently, there are many researches on 3-dimensional geological modeling and visualization, which include 3-dimensional reconstruction and digital geological model, simulation and visualization of geological processes etc. [3-6]. But the 3-dimensional spatial analysis method is mostly used in the field of computer graphics and image processing and these methods focus on the geometric accuracy and efficiency. The existing work includes 3D distance calculation [7-8], 3D superposition analysis [9] and 3D field of search [10] etc. In recent years, scholars in domestic and foreign have made many studies on the 3D images by using mathematical morphology and models, which include the interactive medical image segmentation [11-13], spatial morphological filtering [14-16], the extraction of construction in Radar cloud [17] etc. However, these methods have not been very well applied in metallogenic quantitative prediction in the real three-dimensional geological space. According to the actual need for the spatial morphological analysis of the geogical bodies in stereoscopic quantitative prediction of concealed ore bodies for Fenghuangshan ore field in Tongling, Anhui province, combined with mathematical morphology and Euclidean distance transform theory, a three-dimensional morphological analysis method based on the three-dimensional raster model is presented to gain the shape trend and the shape undulance for the geological body. This method is adaptable to the shape analysis of bend geogical bodies or other complex geological bodies with overlap surfaces, and meets the real three-dimensional requirements of quantitative prediction of concealed ore-body.

\section{Three-dimensional Modelling And Visualization For Geological Bodies}

\section{A. Three-dimensional model of the geological body}

Geological bodies have obvious various shapes which could be described by discrete geometric models. But the ore-controlling action of geological bodies is continuous in geological space and the ore-controlling distribution is fit to be represented by the field model.

Geological data include not only coordinate data and sample data which are achieved from exploration engineering, but also geological survey record, geological maps and so on. The data can be chose to construct the geometric model of geological body. For example, the three-dimensional shape of geological body could be inferred by the wire frame model based on geological section maps. The wire frame model has the advantages of simple structure, less storage space and can better reflect the surface structure of spatial objects.

The voxel model divides the model space into a set of regular cubic three-dimensional arrays. Hence, it can be called three-dimensional raster model. With the advantages of hidden location, simple structure and easy operation, the three-dimensional raster model is conductive to the spatial analysis and calculation. The model is also adaptable to represent the spatial continuous distribution field. In this paper, the three-dimensional raster model is selected to express the ore-controlling distribution of geological bodies.

\section{B. Three dimensional visualization of geological bodies}

3-dimensional geological modeling (3DGM) technology makes it possible to simulate the 3-dimensional geological objects visually such as strata, structures, magmatic bodies, ore bodies and so on. Currently, many three-dimensional geological modeling softwares provide the wire frame modeling functions. In 3D visualization environment, the boundary of geological body could be delineated based on prospecting line profile in exploration and engineering by human-computer interaction. And the 
wire-frame model can be outputted to the three-dimensional raster model by means of block (voxel) division. For instance, the three-dimensional visualization models of Xinwuli magmatic body in Fenghuangshan ore field constructed by the 3DGM software Datamine are shown in Fig. 1. According to the analysis of Metallogenic law for Fenghuangshan ore field, the rock mass is a main ore-controlling geological body. Obviously, the rock mass exits many overlap phenomena in multiple directions.

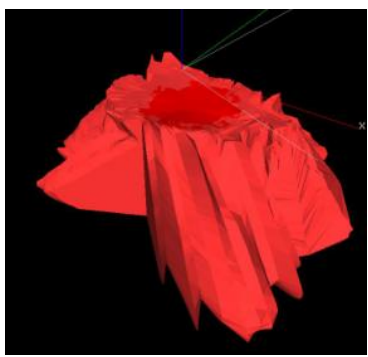

(a) The wire frame model

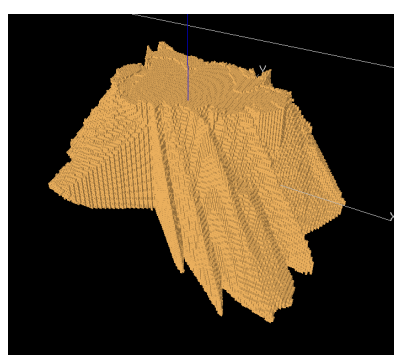

( b ) The raster model

Fig. 1 The 3D visualization model of rock mass

\section{The Shape Analysis Methods For Geological Bodies}

The shape of geological body can be depicted by the geological boundary undulance and the undulance can be conveyed by the relief intensity from geological boundary to its trend surface or lower order trend surface, which reflects the curve changes of geological boundary. Ordinarily, the relief intensity is achieved by trend surface mathematical equations, however, this trend-residue method can not meet the need for the complex shape analysis of geological bodies with overlap surfaces. Based on the 3-dimensinal visualization models of complex geological bodies, a new 3-dimensional shape analysis method is presented by using mathematical morphology and Euclidian distance transform theory and the corresponding shape trend and undulance extraction models are established.

\section{A. The shape analysis Methods Based on Mathematical Morphology}

Mathematical morphology depicts binary image with sets, which includes four basic morphological operations, that is Erosion, Dilation, Opening and Closing. When $\mathrm{A}$ is corroded by $\mathrm{B}$, it can be expressed as $A \Theta B$. A dilated by $\mathrm{B}$ then can be expressed as $A \oplus B$, while $A \circ B$ and $A \bullet B$ can respectively represent Opening and Closing transform.

Suppose that $\mathrm{A}$ is a binary image, $\mathrm{B}$ is a structure element:

$$
\begin{aligned}
& A \Theta B=\{x: b+x \in A\} \\
& A \oplus B=\left[A^{c} \Theta \hat{B}\right]^{c} \\
& A \circ B=(A \Theta B) \oplus B \\
& A \bullet B=(A \oplus B) \Theta B
\end{aligned}
$$

In above formulas, $A^{c}$ is the complementary set of $\mathrm{A}, \mathrm{B}$ rotated $180^{\circ}$ around the coordinate origin is $\hat{B}$.

Geological bodies' boundary expressed by voxel model is a closed surface which is constructed by 3-dimensional discrete voxels. Consequently, it is similar to 3-dimensional binary image, and we could analyze the geological bodies' shape with mathematical morphology theory.

Considering sphere is isotropic in three-dimensional space, the ball structure element is chose to carry out open or close operators on geological bodies. When the ball rolls in the whole three-dimensional space, 
it will include the scroll inside and outside the geological-body boundary. The open operator will take effect when the ball is inside geological-body boundary. The results of an open operator will be a thin shell of the outer boundary formed from the rolling track. While the ball rolling on the insider boundary will scapple the peak on the boundary (in Fig. 2 (b) (c)). Consequently, compared to the original geological boundary, the closed surface has filtered out the peak part which could not be rolled into by the ball. Similarly, the Close operator will take effect when the ball rolls outside geological-body boundary and the scroll will fill up the saddle on the boundary (in Fig. 2 (d) (e)), thereby, an outline with the saddle is gotten. Therefore, combining the two operations on the geological bodies, the geological boundary of the convex peak and concave valley can respectively be scappled and filled so as to get a smooth trend patterns.

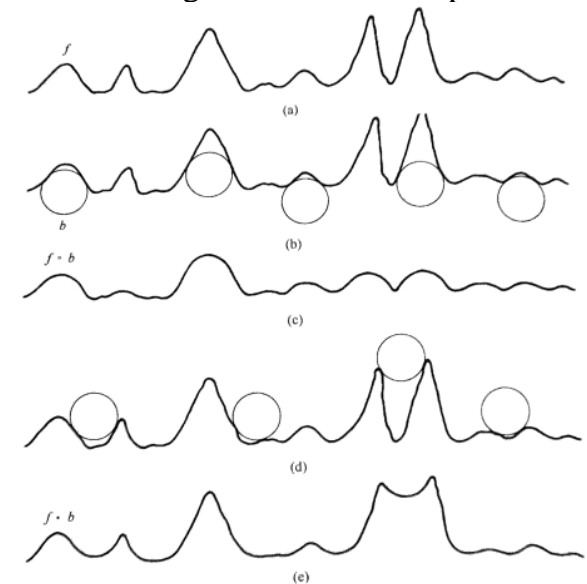

Fig. 2 Opening and Closing operation of spherical structure element on geological body's boundary

Suppose that A is a geological body object, Bball is a spherical structure element, and define the two operations combination as morphological filtering transform:

(1) Opening then Closing filter

$$
\psi(A)=A \circ B_{\text {ball }} \bullet B_{\text {ball }}
$$

(2) Closing then Opening filter

$$
\psi(A)=A \bullet B_{\text {ball }} \circ B_{\text {ball }}
$$

Both of the two transforms can help us get the shape trend and undulance of geological body. The transformed shape smoothness depends on the spherical element radius. The larger the radius is, the more part of the convex peak and concave valley can be filled, and the smoother the shape is. By set operation, these sets can be obtained: the retained shape trend set, the set that constitutes the convex part and the set that fills up the concave part.

\section{B. Euclidean Distance Transform and the Morphological Extraction model}

A new quantitive extraction model for the shape trend and undulance of geological body is presented with the help of Euclidean distance transform technique after mathematics morphological filtering. Referring to the 3-dimensional Euclidean distance transform method based on eight templates proposed by Lin Hong-wei [18], the Euclidean distance field is rendered by scanning all voxels of the raster models, and the concave and convex shape impact on every voxel of the boundary are quantified 
In this method, morphological filtering is carried out once, Euclidean distance transform once, global set operation twice, then the concave and convex attributions and the undulance degree for every voxel can be obtained, the steps are as following:

Step1: Initialize the raster model of the geological body as the 3-dimensional binary image A which is to make shape analysis, define a ball structure element that has the certain radius $r$, the radius can determine the size of the available filtering wave, which can show the undulance degree;

Step2: Take Bball(r) as structure element, and use Opening and Closing operations combination to make morphological filtering on $\mathrm{A}$, then get rock mass shape trend set $\psi(A)$.

Step3: Obtain the convex set $D_{\text {peak }}(A)$ and the concave set $D_{\text {valley }}(A)$ :

$$
\left\{\begin{array}{l}
D_{\text {peak }}(A)=(\psi(A) \cup A)-\psi(A)=A \cap \overline{\psi(A)} \\
D_{\text {valley }}(A)=\psi(A)-(\psi(A) \cap A)=\psi(A) \cap \bar{A}
\end{array}\right.
$$

Step4: By means of Euclidean distance transform, define the Euclidean distance field Eouter outside the set object $\psi(A)$ and the Euclidean distance field Einner inside the set $\psi(A)$.

Step5: For every voxel on geological bodies' boundary, $v \in A$, judge that which set v belongs to:

If $\mathrm{v} \in D_{\text {peak }}(A)$, v belong to the convex, and $\mathrm{v} \in \overline{\psi(A)}$, turn Step6;

If $\mathrm{v} \in D_{\text {valley }}(A)$, v belong to the concave, and $\mathrm{v}_{\in} \psi(A)$, turn Step7;

Otherwise, $\mathrm{v}$ would not belong to geological bodies' boundary.

Step6: Calculate the distance field value Eouter and turn Step5. The value on the voxel v can illustrate the degree of local convex.

Step7: Calculate the distance field value Einner and turn Step5. The value on the voxel v can illustrate the degree of local concave.

\section{Expriment}

The above method is applied to the shape analysis of Xinwuli magmatic body in Fenghuangshan ore field for stereoscopic quantitative prediction of concealed ore bodies. In the light of the analysis of the ore-formation law in Fenghuangshan ore field, Xinwuli magmatic body and its shape undulance play an important role in controlling the distribution of ore body. Based on the 3-dimensioanal raster model shown in fig.1, the aforementioned 3-dimentional morphological analysis method for geological bodies and the morphological extraction model are adopted to extract the shape trend and undulance of Xinwuli magmatic body. Finally, the quantitative simulation of the shape undulance is achieved by the Euclidean distance field model. The models are solved by computer program.

Fig. 3 shows the shape trend with the radius of $400 \mathrm{~m}$ ball structure element by taking Opening and Closing filtering on the basis of 3-dimensional raster model of Xinwuli magmatic body. Fig. 4 shows the extraction model of the concave and convex parts of the shape undulance for the rock mass. Fig. 5 shows the quantitative model (from $-200 \mathrm{~m}$ to $-700 \mathrm{~m}$ elevation) of the shape undulance factor, that is the Euclidean distance field model for the concave and convex parts by 3 -dimensional Euclidean distance transformming . 


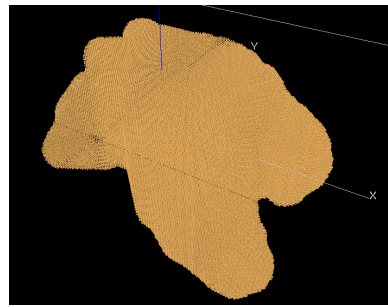

Fig. 3 The The shape trend of Xinwuli magmatic body

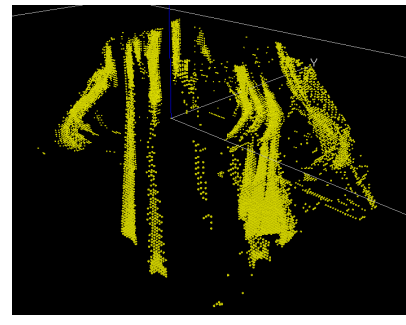

(a) the concave parts

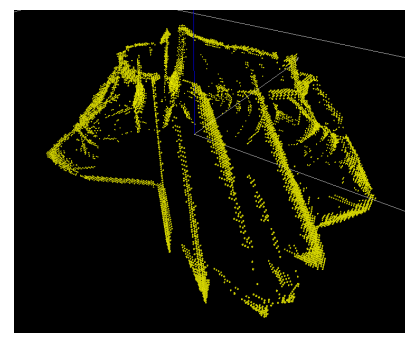

( b ) the convex parts

Fig. 4 extracted the concave and convex parts of the shape undulance

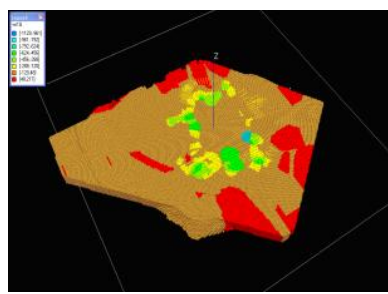

Fig. 5 The quantitative model (from $-200 \mathrm{~m}$ to $-700 \mathrm{~m}$ elevation) of the shape undulance factor

\section{Conclusions}

With the complex shape analysis of the ore-controlling geological bodies with overlay surfaces in stereoscopic quantitative prediction of concealed ore bodies, a new 3-dimensional morphological analysis method is proposed by applying mathematical morphology and Euclidean distance transform theory in this paper. Based on the 3-dimensional discrete raster models of geological bodies in the real 3-dimensional visualization environment, the corresponding morphological analysis algorithm and mathematical model are established. This method is applied to the morphological analysis for XinWuli magmatic body in Fenghuangshan ore-field in Tongling, Anhui province. The shape trend and shape undulance are extracted for the purpose of achieving the geological ore-controlling factor. The trend extraction avoids the trend surface reconstruction for the complex geological body, which is not adaptable to be analyzed by the traditional trend-residue method. By dint of mathematical morphology filtering, when the radius of ball structural elements is changed, a further Hierarchical extraction of the shape undulate classification would be taken by only several times of Opening and Closing filtering operations.

The 3-dimensional morphological analysis method for the complex geological bodies provides a way to extract geometric parameters of geological bodies for implementing the quantification extration of ore-controlling factor. Therefore, it is very significant for the quantitative prediction of concealed ore bodies in 3-dimensional space. 


\section{References}

[1] ZHAO Peng-da. Three-Component Quantitative Resource Prediction and Assessments: Theory and Practice of Digital Mineral Prospecting [J]. Earth Science-Journal of China University of Geosciences, vol. 27, no. 5, pp. 482-489, 2002. (in Chinese).

[2] MAO Xian-cheng, ZOU Yan-hong, LU Xiao-qin, et al. Quantitative analysis of geological ore-controlling factors and stereoscopic quantitative prediction of concealed ore bodies [J]. J Cent South Univ Technol, vol. 16, no.6, pp. 987-993, 2009.

[3] Simon W. H, 3D geoscientific modeling computer Technique for geological characterization [M], 1994, South Sea Int Press Ltd., Hongkong.

[4] Wu Li-xing. Topological relations embodied in a generalized tri-prism (GTP) model for a 3D geoscience modeling system [J]. Computers \& Geosciences, vol. 30, no. 4, pp. 405-418, 2004.

[5] Gong Jian-ya, Cheng Peng-gen and Wang Yan-dong. Three-dimensional modeling and application in geological exploration engineering [J]. Computers \& Geosciences, vol. 30, no. 4, pp. 391-404, 2004.

[6] Jing Ming, Mao Pan, Qu .Hong-gang, et al. GSIS: A 3D geological multi-body modeling system from netty cross-sections with topology [J]. Computers \& Geosciences, vol. 36, pp. 756-767, 2010.

[7] Sud A, Govindaraju N, Gayle R, Manocha D. Interactive 3D distance field computation using linear factorization[C]. Proceedings I3d 2006 - ACM SIGGRAPH Symposium on Interactive 3D Graphics and Games. US A: Association for Computing Machinery, 2006, pp. 117-124.

[8] Jones M W, Baerentzen J A, Sramek M. 3D distance fields: A survey of techniques and applications [J]. Transactions on Visualization and Computer Graphics, vol. 12, no. 4, pp. 581-599, 2006.

[9] Sakuragi F, Nakamoto M, Sasama T, Nakajima Y, Sato Y, Tamura S. System simulator for structural description and error analysis of multimodal 3D data integration systems [J]. Electronics and Communications in Japan, vol. 90, no. 8, pp. 325-338, 2007.

[10]Zuliansyah M, Supangkat S H, Priyana Y, Machbub C. 3D topological relations for 3D spatial analysis[C]. 2008 IEEE International Conference on Cybernetics and Intelligent Systems, CIS 2008. USA: Inst. of Elec. and Elec. Eng. Computer Society, 2008.

[11]Fetita C, Brillet P-Y, Prêteux F J. Morpho-geometrical approach for 3D segmentation of pulmonary vascular tree in multi-slice CT[C]// Pluim J. P. W, Dawant B. M. Medical Imaging 2009 - Image Processing. US A: Proc. SPIE, 2009.

[12] Felix J H S, Cortez P C, Holanda M A, Albuquerque V H C, Colaco D F, Alexandria A R. Lung and chest wall structures segmentation in CT images[C]. Proceedings of VIPIMAGE 2007 - 1st ECCOMAS Thematic Conference on Computational Vision and Medical Image Processing. Netherlands: Taylor and Francis/Balkema, pp. 291-294, 2008,

[13] Naegel B. Using mathematical morphology for the anatomical labeling of vertebrae from 3D CT-scan images [J]. Computerized Medical Imaging and Graphics, vol. 31, no. 3, pp. 141-156, 2007.

[14] Kiwanuka F N, Ouzounis G K, Wilkinson M H F. Surface-area-based attribute filtering in 3D[C]. Mathematical Morphology and Its Application to Signal and Image Processing. Netherlands: Springer Berlin / Heidelberg, pp. 70-81, 2009.

[15] Tankyevych O, Talbot H, Dokladál P, Passat N. Spatially-variant morpho-hessian filter: Efficient implementation and application[C]. Mathematical Morphology and Its Application to Signal and Image Processing. Netherlands: Springer Berlin / Heidelberg, pp. 137-148, 2009.

[16] Li Yong, WU Hua-yi. Filtering airborne LIDAR data based on morphological gradient [J]. Journal of Remote Sensing, vol. 12, no. 4, pp. 633-639, 2008. (in Chinese).

[17] TAO Jin-hua, SU Lin, LI Shu-kai. Method of extracting building model from lidar point cloud [J]. Infrared and Laser Engineering, vol. 38, no. 2, pp. 340-345, 2009. (in Chinese).

[18] LIN Hong-Wei, WANG Guo-Jin. Three dimensional signed Euclidean distance transform and its applications [J]. Chinese Journal of Computers, vol. 26, no. 12, pp. 1645-1651, 2003. (in Chinese). 